\title{
Ultrasonic Method for Identifying Oil Types and Their Mixtures
}

\author{
MAHMOUD SAID RASHED ${ }^{1,2^{*}}$, JOZSEF FELFOLDI ${ }^{1}$
}

\begin{abstract}
The study focused on the efficacy of ultrasonic method for identifying vegetable oils and their mixtures in the formulation of frying oil and its ability in authentication of virgin olive oil. The ultrasonic propagation properties (velocity and Time of Flight (TOF)) were used to classify oil samples and their mixtures at $1 \mathrm{MHz}$. The results revealed the ability to classify oil types in terms of their level of un-saturation, besides it is to identify oil mixtures. Each oil sample could be grouped into different clusters using ultrasonic parameters. Hence, ultrasonic could be used to discriminate the vegetable oil types and their mixtures effectively as a rapid and continuous method in the industrial in-line quality control system of vegetable oils and their mixtures.
\end{abstract}

Keywords: ultrasonic, vegetable oils, Time of Flight (TOF), oils mixtures

\section{Introduction}

Ultrasound is a non-invasive technique and is thus potentially suitable for monitoring the progress of industrial processes in real time. In the literature, many applications can be found regarding the use of ultrasound in different types of products, ranging from solid to liquid materials (Benedito, 2002). Composition assessment by using ultrasonic has been widely reported in the literature. The solid fat content has also been ultrasonically assessed because it has an important implication on the texture, spreadability, and consistency of many materials such as margarine or butter (Coupland \& Mcclements, 1997)

Benedito et al. (2007) mentioned that velocity was the ultrasonic parameter used in most of the aforementioned studies. Velocity can be related to the physicochemical properties of the analyzed materials, like- for example-; composition or structure, and can be used for their analysis. Moreover, Ali and Ali (2014) revealed that the ultrasonic velocity depends on the percentage of unsaturated fatty acid (UFA) and saturated fatty acid

\footnotetext{
* Corresponding author. E-mail: mahmoudsaidrashed88@gmail.com

${ }^{1}$ Department of Physics and Control, Faculty of Food Science, Szent Istvan University, 14-16 Somloi str., 1118 Budapest, Hungary

${ }^{2}$ Food Science and Technology Dept., Faculty of Agric., Alex. Univ., 21545, ElShatby, Alexandria, Egypt
} 
(SFA) contained by the various edible oils. In this respect, ultrasonic velocity has been measured to determine the chemical structure of different oils including the chain length and degree of unsaturation. Therefore, velocity measurements can be used to assess oil composition and adulteration Coupland \& Mcclements (1997). In addition to all that above mentioned, The experiment of Pal et al., ( 2004) was successful in using ultrasonic velocimetry to monitor and study the crystallization process of fats and the results confirmed by Martini et al. (2005). Besides that both of the papers confirmed the specific relationships exist between the ultrasonic velocity and Solid Fat Content (SFC) that enable the measurement of SFC during crystallization; Martini et al. (2005) recommended this technology to be used to perform on-line measurements.

In terms of the frequency range studied, it can be concluded that these edible oils responded better at 1 and $2 \mathrm{MHz}$ frequencies than at 3 and $5 \mathrm{MHz}$. Perhaps the ultrasonic velocity at 1 and $2 \mathrm{MHz}$ may be taken as base values and can be used to detect any adulteration component if these pure oils are adulterated. Velocity has also been correlated to rheological properties of edible oils (castor, olive, groundnut, sunflower, and rapeseed) Benedito et al. ( 2007).

As a result of ultrasonic was becoming an increasingly popular tool for characterizing fatty materials as a physical and non-destructive method (Wassell et al., 2010). The benefits of the fats and oils industry are substantial. On-line sensors can give manufacturers better control over product composition during processing which leads to improved product quality and reduced manufacturing costs. In addition, ultrasound can be used to provide valuable information about the fundamental physicochemical properties of fats and oils. The application of ultrasound in this area will continue to grow. The aim of this work was to evaluate, whether measurements of ultrasonic wave propagation characteristics as a rapid, reliable and fully automated method can be used in-line quality control measurements for classifying oil types and formulating frying oils mixtures.

\section{Material and Methods}

\section{Oil samples and preparation}

Two main groups of oils were collected from local markets of Budapest, Hungary were examined. These groups are classified according to the level 
of unsaturation of the oils to Mono Unsaturated and Poly-Unsaturated fatty acids containing oils.The monounsaturated group contained virgin olive oil, pomace olive oil, and high-oleic sunflower oil; on the other hand, the Polyunsaturated group contained corn oil, soybean oil, and sunflower oil. All oil samples were kept at $7^{\circ} \mathrm{C} \pm 1$ until the time of the analysis. High oleic frying oil mixtures were tested at different high oleic sunflower oil and soybean oil ratios: 0:100, 25:75, 50:50, 75:25 and 100:0 percentages. Each sample was tested in 4 to 6 replicates to ensure the statistical reliability.

\section{Instrumental setup}

The setup of the system used to study the relationship between oil types and ultrasonic parameters is shown in Figure 1. This figure shows the two ultrasonic transducers operating in contact mode (no air between the transducers and the sample). The crystallization cell was designed with two polypropylene windows where the transducers were placed. The distance between two transducers was accurately measured and it was $62.55 \mathrm{~mm}$. Windows were made of polypropylene since this material has minimal effect on ultrasonic wave propagation. A good contact between the transducers and the windows was achieved by means of vacuum grease. Both transducers were aligned so that one of the transducers generated by the ultrasonic wave and the other one received it (transmission mode). The temperature was measured during the analysis using a temperature sensor ICs analog circuit with voltage output connected to a digital system for measuring the temperature as a function of the voltage output.

\section{Ultrasonic measurements}

For measurement of the ultrasonic wave propagation properties, ULTRAN WD50-1 piezoelectric transducers were applied (broadband Dry Coupling Direct Contact sensors of $1 \mathrm{MHz}$ center-frequency). A Vellemann PCSGU250 computer controlled Function Generator and Oscilloscopes were used as pulser and receiver. 


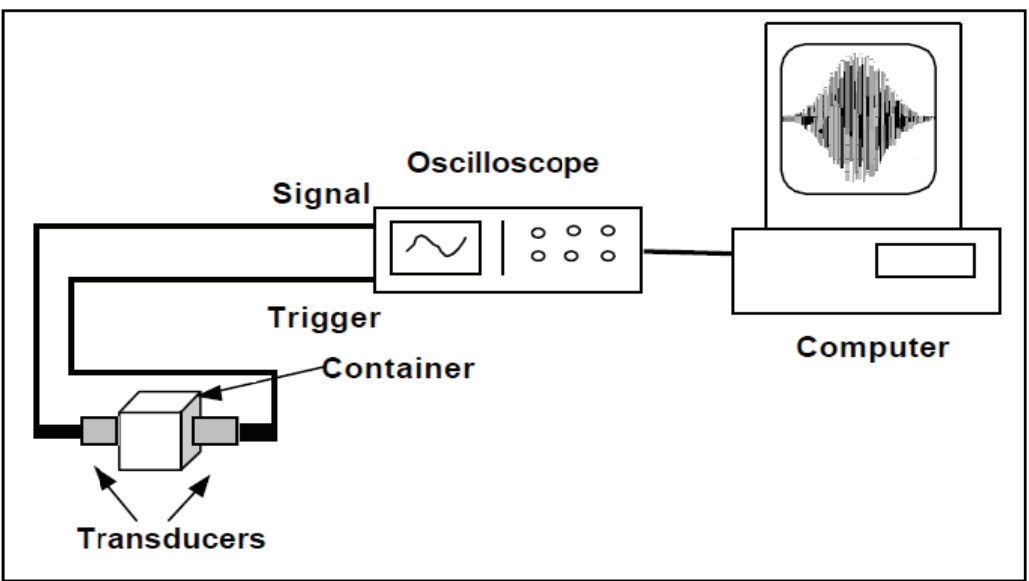

Figure 1. Ultrasonic setup for the measurements of oil samples

Due to the attenuation and dispersion of the investigated material, the simplest transmit signal (a single pulse of appropriate width) results in a noisy, low level received signal of distorted shape, so the determination of the Time-Of-Flight (TOF) is uncertain or impossible. To increase the sensitivity and accuracy of the TOF-detection, a special waveform of more characteristic - conclusively more easy to recognize - the pattern was used: a "chirp" signal of increasing frequency between 0 and $2 \mathrm{MHz}$, modulated by a half sine wave, as it is shown in Figure 2. It has a well-determined spectrum with the maximum at $1 \mathrm{MHz}$ (center frequency of the transducer) Figure 3.

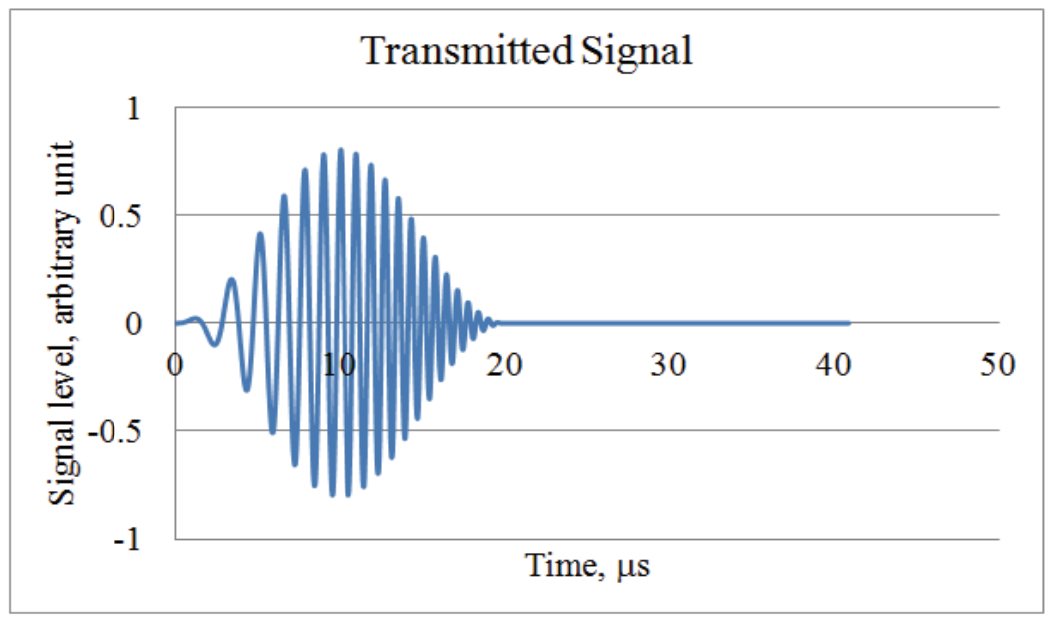

Figure 2. Chirp signal (time domain) 
The time delay was calculated by the cross-correlation of the transmitted and received signals. It was determined by the following equation:

Where

$$
\operatorname{Cross} \operatorname{Corr}(t)=\operatorname{IFFT}(\overline{\overline{F F T}(\mathrm{U} 1)} \cdot \operatorname{FFT}(\mathrm{U} 2))
$$

- $\quad \mathrm{U} 1$ and $\mathrm{U} 2$ are the transmitted and received signals, respectively

- $\overline{F F T(U 1)}$ is the complex conjugate of the Fast Fourier Transformed value of $U 1$

- $\quad$ FFT(U2) is the Fast Fourier Transformed value of U2

- $\operatorname{IFFT}()$ is the inverse Fourier transform

CrossCorr $(t)$ is a time-domain function, and its maximum corresponds to the TOF-value (the maximal similarity between the transmitted and received signals).

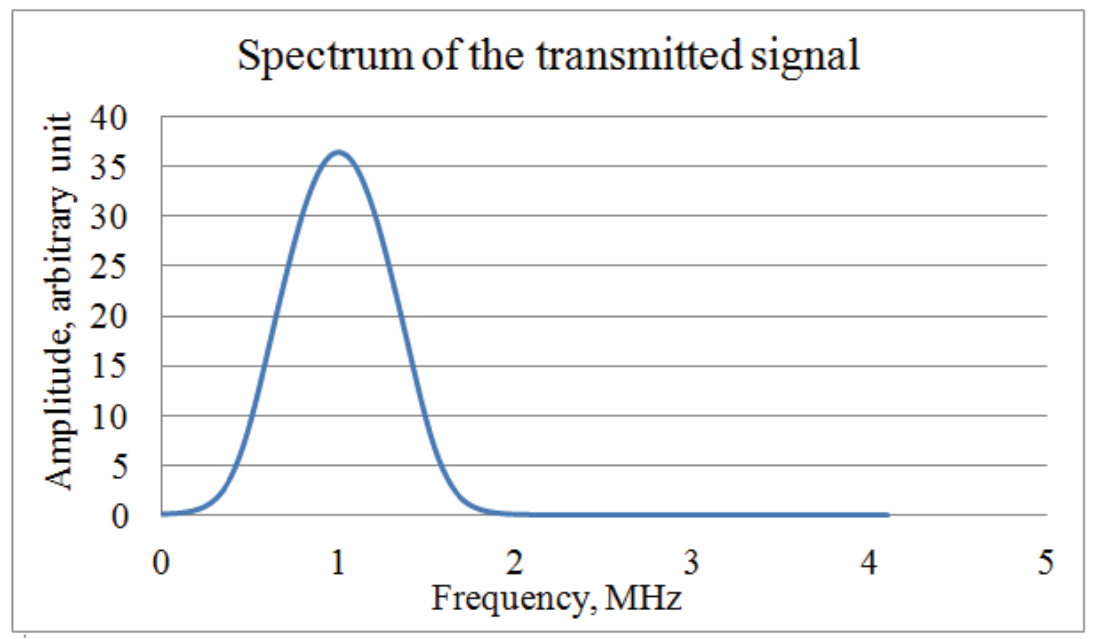

Figure 3. Typical chirp Signal (frequency domain)

\section{Statistical analysis}

Statistical design of the whole experiment was carried out using a completely randomized design (CRD). It was used for evaluating the effect of the level of unsaturation to the ultrasonic velocity and time of flight. The results are presented as means of four replicates with $95 \%$ confidence intervals for means. The coefficient of determination was determined by the 
percentage of mixing high oleic sunflower oil and time of flight. The results are presented as means of six replicates with standard deviations.

\section{Results and Discussion}

\section{A - Temperature Dependency}

Figure 4 shows the temperature dependency for sunflower oil. In the legend of Fig. 4 "up 1 \& 2" are representing repetitions for the measuring TOF while heating up the oil for "down as $1 \& 2$ " are representing repetitions for measuring TOF while cooling down the oil.

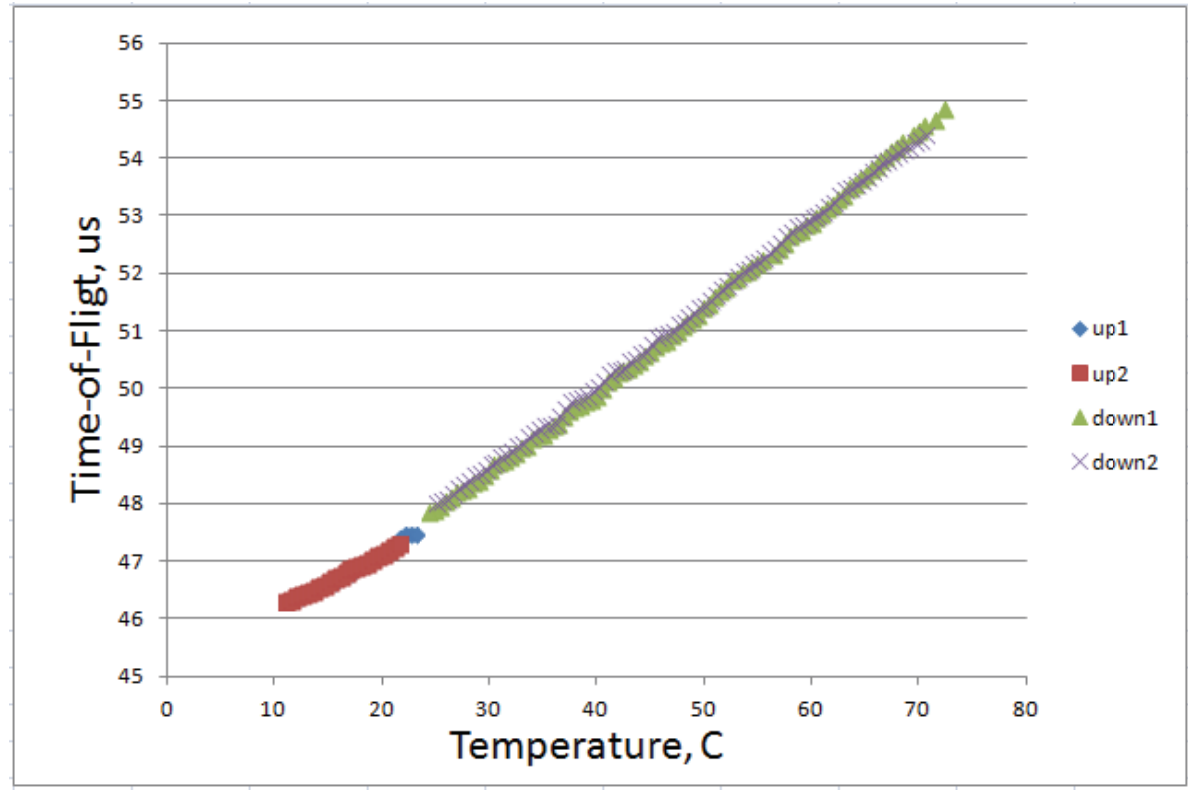

Figure 4. Temperature dependency of Time-of-Flight of ultrasound for sunflower oil.

These results in the experiment have confirmed these data obtained by Wassell et al., ( 2010) as sound velocity determined in rapeseed oil as a function of temperature with a correlation coefficient of 0.997 . The results showed the SFC values as a function of temperature for $30 \%$ palm stearin and $70 \%$ rapeseed oil fat blend. The best correlation $\left(\mathrm{R}^{2}=0.99\right)$ lies in the temperature range of $15-35^{\circ} \mathrm{C}$. Therefore, measurement of ultrasonic 
velocity and time of flight is suitable to use in-line measurements for continuous quality control processes for observing the changes in SFC in oil mixtures.

Further development to validate ultrasonic velocity and TOF measurements by coupling the results with rheology measurement techniques it could see the advantage of these measurements as an essential tool for both industrial in-line process control, and further academic understanding of fat blends structuring.

\section{B - Ability of Classification}

The propagation speeds of ultrasound for the measured oils at $23^{\circ} \mathrm{C}$ are in Table 1. The results revealed that there are significant differences between oil types. The principle of classification was carried out based on the differences between ultrasonic velocities measured were higher than 2 standard divisions of the measurements.

Table 1. The speed of ultrasound propagation in different types of Oils at $\left(23^{\circ} \mathrm{C}\right)$

\begin{tabular}{|c|c|c|}
\hline \multirow{2}{*}{ Oil Types } & Kind of Oil & Ultrasound speed (m/s) \\
\hline \multirow{3}{*}{ Mono Unsaturated } & virgin olive oil & $1436.3 \pm 0.3$ \\
\cline { 2 - 3 } & pomace olive oil & $1437.6 \pm 0.3$ \\
\cline { 2 - 3 } & high oleic sunflower oil & $1438.6 \pm 0.3$ \\
\hline \multirow{3}{*}{ Poly Unsaturated } & corn oil & $1441.8 \pm 0.3$ \\
\cline { 2 - 3 } & soybean oil & $1442.6 \pm 0.3$ \\
\cline { 2 - 3 } & sunflower oil & $1442.3 \pm 0.3$ \\
\hline
\end{tabular}

The measurement of the speed of ultrasound in different oil samples revealed that this measurement is able to classify edible oils and fats according to their degree of unsaturation in two main groups: Mono Unsaturated Fatty Acids (MUFA) oils and Poly Unsaturated Fatty Acids (PUFA) oils. Although the PUFA and MUFA groups are significantly different and MUFA oils can be distinguished within the MUFA group, there were no significant differences between the PUFA oils. Therefore, the speed of ultrasound propagation could be classified as one of the promising techniques for investigating vegetable oils. 


\section{C - The ability of identification frying oil mixtures}

Frankel (1994) mentioned that the aim for formulating frying oil mixtures is preparing more stable vegetable oils with a wide range of desired fatty acid compositions by mixing different proportions of High Oleic sunflower oil with Soybean Oil. Figure 5 represents the relationship between the speed of ultrasound and the percentages of high-oleic sunflower oil in frying mixtures. The results revealed that speed of ultrasound is strongly connected with a high correlation $\mathrm{R}^{2}=0.9479$ with the ratios of high-oleic sunflower oil in frying oil mixture components.

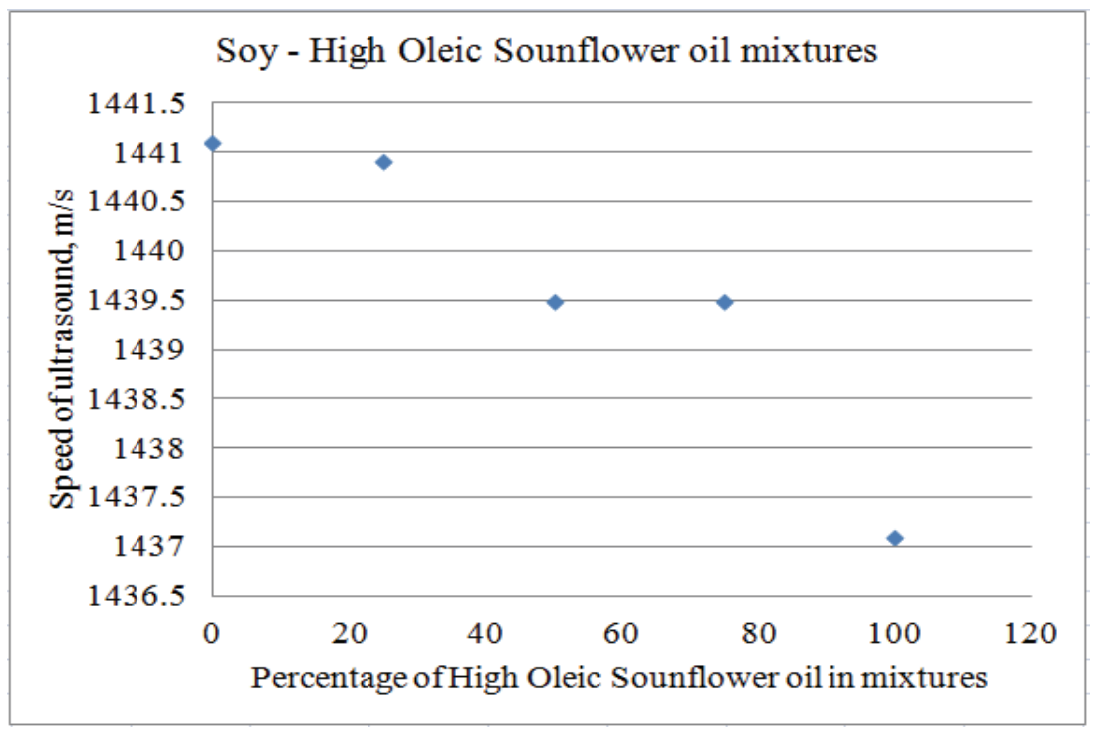

Figure 5. Relationship between the speed of ultrasound at $1 \mathrm{MHz}$ and the percentages of high-oleic sunflower oil in frying mixtures

These results confirmed Ali and Ali (2014) findings that ultrasonic velocity depends on the $\%$ of UFA and SFA contained by the various edible oils. Moreover, the ultrasonic velocity at $1 \mathrm{MHz}$ may be taken as the base values and can be used to detect any adulteration component if these pure oils are adulterated. Therefore, an ultrasonic method is suitable for continuous quality inspection system in formulating frying oil mixtures. 


\section{Conclusion}

Variation of ultrasonic velocity and TOF with temperature in high viscous vegetable oils is one of the effective physical measurements in vegetable oils industries. It is observed that ultrasonic velocity of vegetable oils decreases with the increase of temperature. Therefore, the method is giving the possibilities for predicting the time of flight at a given temperature. Velocities of sound in various vegetable oils vary based on the composition of fatty acid and degree of saturation of oils. Moreover, ultrasound velocity measurement is a sensitive method for detecting the changes in the oil mixtures composition significantly. Although the results obtained needs more investigations for further generalization of the usage of the speed of ultrasound propagation for vegetable oils as one of the sensitive methods for investigating oil in industrial in-line process control of oil blending systems.

\section{References}

Ali, S. M., \& Ali, B. (2014). Attenuation of Ultrasound in Commonly used Vegetable Oils at Low Frequencies. International Journal of Science, Environment, and Technology, Vol. 3, No 5, 2014, 1803 - 1809.

Benedito, J., Mulet, A., Velasco, J.and Dobarganes, M. (2002). Ultrasonic Assessment of Oil Quality during Frying. J. Agric. Food Chem, Vol. 50, No 16, 2002, 4531 - 4536. http:/ / doi.org/10.1021/jf020230s

Benedito, J., Dobarganes, M. C., Mulet, A., \& Garc1, J. V. (2007). Rapid evaluation of frying oil degradation using ultrasonic technology. Food Research International, Vol. 40, No 3,2006,406-414. http:/ / doi.org/10.1016/j.foodres.2006.10.017

Coupland, J. N., \& Mcclements, D. J. (1997). Physical Properties of Liquid Edible Oils. JAOCS, Vol. 71, No 3,1997, 255-259.

Martini, S., Bertoli, C., Lidia, M., Neeson, I., \& Marangoni, A. (2005). In situ Monitoring of Solid Fat Content by Means of Pulsed Nuclear Magnetic Resonance Spectrometry and Ultrasonics. JAOCS , Vol.82, No 5,2005, 305-312.

Pal, A., Mcclements, D. J., \& Marangoni, A. G. (2004). Solid fat content determination by ultrasonic velocimetry.Food Research International, Vol.37, No 6,2004, 545-555. http:/ / doi.org/10.1016/j.foodres.2003.12.010

Wassell, P., Wiklund, J., Stading, M., Bonwick, G., Smith, C., Almiron-roig, E., \& Young, N. W. G. (2010). Original article Ultrasound Doppler based in-line viscosity and solid fat profile measurement of fat blends. International Journal of Food Science \& Technology, Vol.45, No 5,2010, 877-883. http://doi.org/10.1111/j.13652621.2010.02204.x 